\section{Psychological reality of physical concepts ${ }^{1}$}

PAUL E. JOHNSON, DAVID L. COX, and THOMAS E. CURRAN, ${ }^{2}$ University of Minnesota, Minneapolis, Minn. 55455

Fifty college physics students rated the similarity of pairs of concept words in analytical mechanics, and also provided 1 min of continued word associations to each individual concept word. The mean proportion of responses in common on the word association test was used as an index of associative similarity among concepts. Mean rating-scale judgments served as an index of perceived similarity. These two indices were interpreted as proximity measures and were scaled, using multidimensional scaling procedures with both a Euclidean and a city-block metric. Results suggest that either a two-dimensional configuration with a Euclidean metric or a three-dimensional configuration with a city-block metric describes the underlying structure of the similarity relations. The three-dimensional configuration correlated well with an hypothesized geometric model.

By the psychological reality of physical concepts, we mean how representations of these concepts are perceived and evaluated. In order to study physical concepts in this sense, we conceive of a psychological space with a set of points in this space corresponding to perceived relations among the concepts.

The physical concepts we propose to describe in this fashion are drawn from the subject matter of analytical mechanics. They are power, work, force, momentum, acceleration, and velocity. These concepts are defined by mathematical equations which ultimately specify some relationship between mass, distance, and time, which are in turn defined by things such as the balance, the meter stick, and the clock.

The formal or logical relationships among the first six concepts above can be conceptualized by means of a geometric model derived from their mathematical definitions. ${ }^{3}$ This model is presented in Fig. 1.

Because each concept in Fig. 1 can be written in terms of the operations of integration and differentiation with respect to some combination of mass $(\mathrm{m})$, distance $(x)$, and time $(t)$, they can be plotted as points in the space generated by the model. To plot concepts in this fashion, we assume that each differentiation or integration represents one unit and that the concept of distance constitutes the origin of the space. The point immediately to the right of the origin is then written as $\int x d m$, and the point immediately below the origin is $\mathrm{dx} / \mathrm{dt}$. Our procedure not only allows the six chosen concepts to be located in the space (e.g., $\mathrm{dx} / \mathrm{dt}=$ velocity) but also permits a representation of points that are not readily labeled by concept words in mechanics (e.g., $\left.\int x \mathrm{dm}\right)$. Notice, however, that the six concepts are distributed so that four of them lie in the mass-time plane. Experimentally this may be an unfortunate artifact of the way physicists have labeled concepts with words. On the other hand, such labeling may tell us something about the scientist's conception of his discipline. At any rate, the scheme in Fig. 1 permits other concepts to be studied, provided we describe these concepts in a language such as mathematics.

The model in Fig. 1 suggests that distances between the points in the space can be used to predict obtained similarity relations among representations of these points. Thus, the closer two concepts are in Fig. 1, the greater their logical similarity; and we would expect that the greater the logical similarity, the greater would be their perceived similarity in appropriate tasks. Put another way, our approach is based upon the assumption that the study of internal representations of physical concepts must focus upon the relationship between the structure among external representations of these concepts and the structure among their corresponding intemal representations (cf. Shepard \& Chipman, 1970).

\section{METHOD}

In the present study, similarity relations among the six concept words were indexed in two ways, one direct and one indirect. The direct measure was obtained by

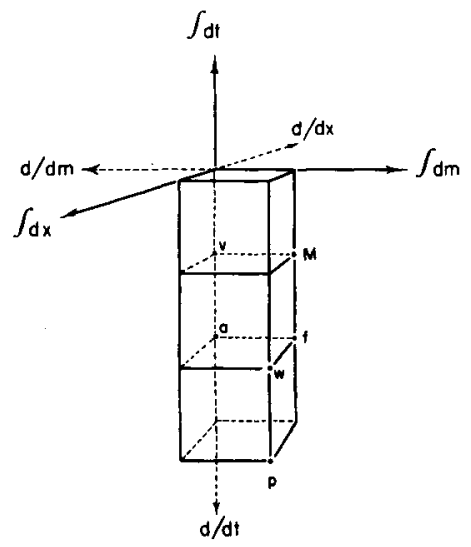

Fig. 1. Geometric model of selected concepts in analytical mechanics. presenting Ss with the 15 possible pairs of the six concepts and asking them to judge on a 7-point rating scale how similar they felt these pairs were to one another. The indirect measure of similarity was based upon a word association test. Here Ss were given the six concepts and instructed to write as many words as they could for each concept in $1 \mathrm{~min}$.

The tasks were presented to $S s$ in the form of a booklet containing the word association test (WA) and the similarity rating test (SR), in that order. The six concept words were randomized for each $S$ on the WA test. In the SR test, pairs of the six words were presented in two random orders, each word appearing equally often on both sides of the rating scale in each random order.

Ss were 50 physics majors at the University of Minnesota. Each $S$ received $\$ 3.00$ for serving in the experiment. RESULTS AND DISCUSSION

Two dependent measures were used: mean rating scale judgments between pairs of words in the SR test and mean proportion of associative responses each pair of concept words had in common on the WA test.

These data were treated as proximity measures and served as input to a multidimensional scaling program based upon the nonmetric scaling procedure developed by Shepard $(1962 a, b)$ and modified by Kruskal (1964a, b). This program subsequently generated a psychological space for the six concepts in each task. Both Euclidean and city-block distance metrics were used in computing these scaling solutions.

While the pervasiveness of the Euclidean spatial model makes the Euclidean distance metric a reasonable one to use for interpreting similarity relations, there are data to suggest that, in some cases, the city-block metric is more appropriate (Attneave, 1950; Hyman \& Well, 1967). Moreover, because our geometric model is one in which the diagonal distances have no apparent mathematical interpretation (one must always perform the operations of differentiation and integration separately; they cannot be done simultaneously), there is reason to expect the underlying metric of the model to be city block.

To establish the reasonableness of the scaling solutions, a measure was utilized which indexes the goodness of fit of each solution to the proximity data. This goodness-of-fit measure is called "stress," and a good fit is indicated by low stress. Stress indices for solutions in one, two, and three dimensions, based upon both Euclidean and city-block distances, appear in Table 1 . 
Table 1

Stress Measures for Multidimensional Scaling Solutions in One, Two, and Three Dimensions

\begin{tabular}{lccccr}
\hline & \multicolumn{2}{c}{ Word Association } & & \multicolumn{2}{c}{ Similarity Rating } \\
\cline { 2 - 3 } Dimensions & Euclidean & City Block & & Euclidean & City Block \\
\hline One & .086915 & .086915 & .129602 & .129602 \\
Two & .000000 & .000408 & .000001 & .055526 \\
Three & .000000 & .000000 & .000000 & .000000 \\
\hline
\end{tabular}

The stress in three dimensions is "perfect" (i.e., zero) in all cases, according to Kruskal's (1964a) criteria. Low stress in two dimensions (particularly with the Euclidean metric) may be the result of having only six data points, since, as mentioned earlier, four of the six points in Fig. 1 lie on a plane. However, the two-dimensional solution does have an interpretation and, for this reason, it is presented (with Euclidean distances) in Fig. 2.

The six points in Fig. 2 are divided into two groups by the vertical axis, such that those points to the right of this line (momentum, force, work, and power) all involve the concept of mass, while those to the left (acceleration and velocity) do not. This division of concepts parallels a division of the subject matter of mechanics into dynamics and kinematics.

The configuration of points in Fig. 2 is also divided into two groups by an horizontal axis. Those concepts below the axis (velocity and momentum) involve uniform motion, while those above this line (acceleration, force, work, and power) involve accelerated motion. There is a similar interpretation, although not an identical configuration, for the two-dimensional solutions with city-block distances.

Because the two-dimensional solutions with Euclidean distances have essentially zero stress, the three-dimensional Euclidean solutions may be nonsingular

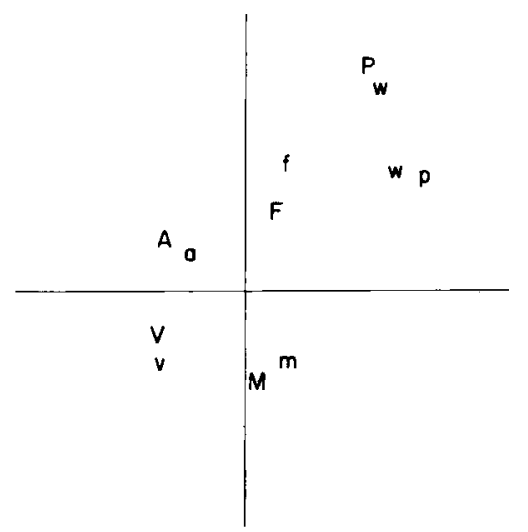

Upper Case, SR Test

Lower Case, WA Test

Fig. 2. Scaling solution in two dimensions with Euclidean distance. (cf. Shepard, 1962b, 1966). Moreover, with only six data points, the likelihood of finding zero stress by chance is good (Klahr, 1969). However, in the case of city-block distance, the stress is zero in three dimensions but not in two dimensions. (It should be noted that, as yet, there is no procedure for determining "significantly different" from zero). Therefore, the three-dimensional solution with city-block distance may also be a representation of the underlying structure of the proximity data.

The rank-order correlations between distances in the geometric model and distances in the three-dimensional scaling solution for the city block metric were .90 for the WA test and .85 for the SR test $(\mathrm{p}<.01$ in both cases). The correlation between scaled distances on the WA and SR test was $.87(p<.01)$. In the case of Euclidean distance, these correlations were $.90(\mathrm{p}<.01), .85(\mathrm{p}<.01)$, and .89 $(p<.01)$, respectively.

Correlations between relative distances are a simple means of comparing different and the distances between points in a geometric model. While alternative procedures have recently become available (cf. Carroll, 1968; Carroll \& Chang, 1969), we feel that the magnitude of the above correlations warrants the conclusion that the psychological spaces produced by scaling solutions in three dimensions for the two tasks are reasonably similar to one another. This suggests that the SR and WA tasks, although quite different, are in some respects accessing the same underlying structure. Moreover, the magnitude of the correlation between scaled and logical distances tentatively supports the proposition that the geometric model describes this structure.

While the two-dimensional scaling solution of the proximity data can be interpreted, we regard the three-dimensional solution as a more parsimonious explanation of the obtained similarity relations because (1) it is in agreement with our a priori model for the underlying structure of these relations (cf. Klahr, 1969), and (2) it has the two-dimensional interpretation) of generating a large variety of potential stimulus points. whether a given stress value is scaling solutions or one scaling solution capability (not inherent in the
In the present study, our stimulus points were limited to those that had verbal labels in mechanics. With words as stimuli, the underlying dimensions are not likely to be clear or compelling. Therefore, on grounds suggested by Torgerson (1958), the Euclidean metric is the most reasonable way of accounting for our data. However, we feel the model in Fig. 1 is more readily interpreted using the city-block metric. Unfortunately, the six points in Fig. 1 lie such that the rank order of interpoint distances is virtually the same, regardless of metric $(r=.99)$. As a result, we cannot make an empirical assessment as to which distance metric is more appropriate.

As a final test of our model, rank order correlations were computed between initial proximity measures for each task and logical distances using both the city block and Euclidean metric. These correlations were .90 for the WA task and .83 for the SR task with the city block metric and .91 and .83 , respectively, with the Euclidean metric ( $p<.01$ in all cases). There is considerable similarity between these correlations and those obtained between the logical distances and scaled distances, thus substantiating the hypothesis that the three-dimensional solution does describe the underlying structure of the original proximity data.

Because the present study utilized only six stimulus points from Fig. 1, our results must be interpreted with caution. Additional studies using a greater number of points are needed and metric scaling should be used as a check on nonmetric procedures. Finally, a word is in order regarding the notion of similarity which is at the heart of this study. Since our stimulus points represented rather complex concepts, subsequent work may require a distinction between similarity as a multidimensional stimulus attribute and similarity as a derived cognitive relation (Torgerson, 1965).

\section{REFERENCES}

ATTNEAVE, F. Dimensions of similarity American Journal of Psychology, 1950, 63, 516-556.

CARROLL, J. D. Generalization of canonical correlation analysis to three or more sets of variables. Reprinted from the Proceedings, 76th Annual Convention, APA, 1968, 227-228.

CARROLL, J. D., \& CHANG, J. J. A new method for dealing with individual differences in multidimensional scaling. Unpublished report from The Bell Telephone Laboratories, Murray Hill, New Jersey, 1969.

HYMAN, R., \& WELL, A. Judgments of similarity and spatial models. Perception \& Psychophysics, 1967, 2, 233-248.

KLAHR, D. A Monte Carlo investigation of the statistical significance of Kruskal's nonmetric scaling procedure. Psychometrika, 1969, 34, 319-330.

KRUSKAL, J. B. Multidimensional scaling by 
optimizing goodness of fit to a nonmetric hypothesis, Psychometrika, 1964a, 29, 1-27.

KRUSKAL, J. B. Nonmetric multidimensional scaling: A numerical method. Psychometrika, 1964b, 29, 115-129.

SHEPARD, R. N. The analysis of proximities: Multidimensional scaling with an unknown distance function. 1. Psychometrika, 1962a, $27,125-140$.

SHEPARD, R. N. The analysis of proximities: Multidimensional scaling with an unknown distance function. II. Psychometrika, 1962b, 27, 219-246.

SHEPARD, R. N. Metric structures in ordinal data. Joumal of Mathematical Psychology, $1966,3,287-315$

SHEPARD, R. N., \& CHIPMAN, S. Second-order isomorphism of internal representations: Shapes of states. Cognitive Psychology, 1970, 1, 1-17.

TORGERSON, W. S. Theory and methods of scaling. New York: Wiley, 1958.
TORGERSON, W. S. Multidimensional scaling of similarity. Psychometrika, 1965, 30, 379-393.

\section{NOTES}

1. This work was supported in part by grants to the MINNEMAST center at the University of Minnesota from The National Science Foundation (GE-3), and in part by grants to the University of Minnesota Center for Research in Human Learning from the National Science Foundation (GS-1761), the National Institute of Child Health and Human Development (HD-01136-04), and from the Graduate School of the University of Minnesota.

2. The authors would like to express their appreciation to Roger N. Shepard for his helpful comments on an earlier draft of this paper.

3. power $(p)=d / d t\left(\int \mathrm{md}^{2} \mathrm{x} / \mathrm{dt}^{2} \mathrm{dx}\right)$, work $(w)$ $=\int \mathrm{md}^{2} \mathrm{x} / \mathrm{dt}^{2} \mathrm{dx}$, force (f) $=\mathrm{md}^{2} \mathrm{x} / \mathrm{dt}^{2}$, momentum $(M)=\mathrm{mdx} / \mathrm{dt}$, acceleration (a) $\mathrm{d}^{2} \mathrm{x} / \mathrm{dt}^{2}$, velocity $(\mathrm{v})=\mathrm{dx} / \mathrm{dt}$.

\section{On the asymmetry of the phase-difference function in binocular flicker}

\section{C. CORBALLIS, ${ }^{1}$ McGill University, Montreal, Canada}

Foley and Stager have reported that, for right-eye dominant $S s$, the function relating critical flicker frequency (CFF) to the phase lag of pulses to the left eye behind pulses to the right eye is concave downwards for lags between 0 and 180 deg and concave upwards between 180 and 360 deg. This implies a cyclic function such that maximum and minimum CFFs occur not at but slightly above 0 and $180 \mathrm{deg}$, respectively, which in turn suggests faster processing of information to the left than to the right eye. A possible explanation is that the two cerebral hemispheres contributed differently to the resolution of flicker, with the more important contribution coming from the right hemisphere.

Binocular critical flicker frequency (CFF) varies as a function of interocular phase difference. When the pulses to each eye are exactly in phase (0-deg phase difference), CFF is higher than when they are completely out of phase (180-deg phase difference) (Baker \& Bott, 1951; Kinsbourne \& Coughlin, 1969; Perrin, 1954; Thomas, 1955). There is also evidence that the phase-difference function is asymmetrical. Foley \& Stager (1965) reported two experiments in which each pulse to the left eye was made to lag behind the corresponding pulse to the right eye in 45-deg steps, ranging from 0 to $180 \mathrm{deg}$ in one experiment and from 180 to $360 \mathrm{deg}$ in the other. The data, averaged over six Ss in the first experiment and over five $S s$ in the second, are shown in Fig. 1. It can be seen that the function is concave downwards between 0 and $180 \mathrm{deg}$ and concave upwards between 180 and $360 \mathrm{deg}$. All Ss were female, right-eye dominant, and four of them served as Ss in both experiments. Further details can be obtained from Foley and Stager's original report.

Foley and Stager interpret the asymmetry of the function as evidence that neural impulses from the right eye are given more weight at some central locus than neural impulses from the left eye. Thus the difference in CFF between 0 and $45 \mathrm{deg}$ is rather small, because at $45 \mathrm{deg}$ an impulse from the nondominant left eye arrives during an "on" phase for the dominant right eye, and consequently has little effect. By contrast, the difference between 315 and $360 \mathrm{deg}$ is relatively large, because at $315 \mathrm{deg}$ the left-eye impulse arrives centrally during a right-eye "off" phase, and so has a greater effect in reducing CFF. This account is plausible only if special prominence is given to the neural impulses generated by the onset of light to the left eye, since exactly the opposite argument would hold for neural impulses initiated by the offset of light.

\section{AN ALTERNATIVE \\ INTERPRETATION}

Foley and Stager explicitly reject a suggestion by Sampson (1962) that the phase-difference function depends on differences between the eyes in the time taken centrally to integrate information. According to Sampson, information to the left eye is integrated more slowly than that to the right eye, so that when light pulses are in phase peripherally the corresponding neural impulses are out of phase centrally, and when they are out of phase peripherally they are in phase centrally. This suggestion is implausible on two counts: First, it suggests that CFF is highest when flicker is out of phase centrally, whereas on intuitive grounds, at least, one would expect the opposite; and secondly, it implies that the difference in integration time between the two eyes is equal to the period of one semicycle of flicker, which is improbable. Nevertheless, Foley and Stager's data can be taken as evidence for interocular differences in processing time, though not quite in the manner that Sampson proposed.

The data points in Fig. 1 suggest a cyclic function that is displaced slightly to the right, so that maximum and minimum CFF might be said to occur, not precisely at 0 and $180 \mathrm{deg}$, respectively, but slightly above these values. This is consistent with the notion that left-eye information is processed somewhat more rapidly than right-eye information, and that a slight delay of input to the left eye is therefore necessary to restore symmetry centrally. To examine this idea in more detail, equations of the form $Y=a \cos (X-b)+c$ were fitted to the data for each $S$ in each experiment by the method of least squares. $Y$ represents $C F F$, and $X$ the interocular phase difference. The calculated constants, a, b, and c, are shown in Table 1 , and the continuous curves plotted in Fig. 1 are the averaged curves derived from the fitted equations.

Table 1 indicates that in every case but one the constant $b$ is positive, which can be interpreted to mean that processing of left-eye information was faster than that of right-eye information. The actual time differences may be estimated from the formula

$$
t=\frac{1000 \times b}{360 \times c} \mathrm{msec}
$$

and these values are also shown in Table 1. These time differences could have arisen from two sources. They could reflect a bias in the calibration of the equipment, so that when the setting was at $0 \mathrm{deg}$, for example, the actual interocular phase difference was slightly positive. Alternatively, they could have occurred in the processing of information by the Ss, as suggested above. The fact that the time lags for Ss 1,2 , and 5 were fairly consistent between experiments, compared with the relatively 